\title{
A Family with Axenfeld-Rieger Syndrome: Report of the Clinical and Genetic Findings
}

\author{
Hee Jung Yang ${ }^{1}$, You Kyung Lee ${ }^{1}$, Choun-Ki Joo ${ }^{1}$, Jung Il Moon ${ }^{1}$, Jee Won Mok ${ }^{2}$, Myoung Hee Park ${ }^{1}$ \\ ${ }^{I}$ Department of Ophthalmology, The Catholic University of Korea College of Medicine, Seoul, Korea \\ ${ }^{2}$ Catholic Institute for Visual Science, Department of Ophthalmology and Visual Science, Seoul St. Mary's Hospital, The Catholic \\ University of Korea College of Medicine, Seoul, Korea
}

Purpose: To describe clinical findings in a Korean family with Axenfeld-Rieger syndrome.

Methods: A retrospective review of clinical data about patients with diagnosed Axenfeld-Rieger syndrome. Five affected members of the family underwent a complete ophthalmologic examination. We screened the forkhead box $\mathrm{C} 1$ gene and the pituitary homeobox 2 gene in patients. Peripheral blood leukocytes and buccal mucosal epithelial cells were obtained from seven members of a family with Axenfeld-Rieger syndrome. DNA was extracted and amplified by polymerase chain reaction, followed by direct sequencing.

Results: The affected members showed iris hypoplasia, iridocorneal adhesions, posterior embryotoxon, and advanced glaucoma in three generation. None had systemic anomalies. Two mutations including c.1362_1364insCGG and c.1142_1144insGGC were identified in forkhead box C1 in four affected family members.

Conclusions: This study may help to understand clinical findings and prognosis for patients with Axenfeld-Rieger syndrome.

Key Words: Anterior segment dysgenesis, Axenfeld-Rieger syndrome, Forkhead box $\mathrm{C} 1$ gene

Axenfeld-Rieger syndrome (ARS) is a disorder characterized by anterior segment dysgenesis, including abnormalities in the cornea, iris, and anterior chamber angle structures. It is occasionally associated with systemic developmental abnormalities, including craniofacial anomalies, dental abnormalities, cardiac valvular malformation, periumbilical redundant skin, and variable neurological and skeletal disorders. Patients with ARS have an approximately $50 \%$ risk of developing glaucoma, and this risk is

Received: October 3, 2014 Accepted: November 25, 2014

Corresponding Author: Myoung Hee Park, MD, PhD. Department of Ophthalmology, Yeouido St. Mary's Hospital, The Catholic University of Korea College of Medicine, \#10 63-ro, Yeongdeungpo-gu, Seoul 150-713, Korea. Tel: 82-2-3779-1243, Fax: 82-2-3779-1245, E-mail: marypark@ catholic.ac.kr higher in cases where the peripheral iris is highly inserted into the trabecular meshwork [1-3].

ARS is an autosomal dominant disorder with high penetration. It is primarily caused by a mutation of the pituitary homeobox 2 (PITX2) or forkhead box $\mathrm{C} 1$ (FOXC1) gene. Mutations in PITX2 on chromosome 4q25, or in FOXC1 at $6 \mathrm{p} 25$, have been identified in approximately $40 \%$ to $70 \%$ of patients with ARS $[3,4]$. These genes play an important role in the development of the anterior segment. FOXC1 is a member of the forkhead family of transcription factors, which have an evolutionarily conserved DNA-binding domain consisting of a 110-amino acid motif. It regulates embryogenesis through gene products that participate in the migration and differentiation of mesenchymal cells $[5,6]$. FOXC1 mutations include missense, frameshift, and nonsense mutations, as well as deletions and duplications. 
Among these, point mutations are the most common. Variable phenotypes result, with features of haploinsufficiency or gain-of-function mutations [5].

In this report, we describe the clinical characterization of a family with ARS in three generations and report the association of the genetic etiologic factors with ARS.

\section{Materials and Methods}

A family with ARS was referred to our clinic. There were five affected members of the family in three generations (Fig. 1). The proband in this family was a 19 -year-old male. We performed complete ophthalmologic evaluations of all affected members of the family, including a slit-lamp examination, fundus examination, anterior segment photography, gonioscopic examination, central corneal thickness measurement (US-1800 pachymeter; NIDEK, Gamagori, Japan), visual field test (Humphrey Visual Fields Analyzer 24-2; Carl Zeiss, Jena, Germany), retinal nerve fiber layer (RNFL) thickness measurements (Cirrus HD OCT, Carl Zeiss Meditec, Dublin, CA, USA), and axial length measurement (optical biometer IOL Master, Carl Zeiss Meditec).

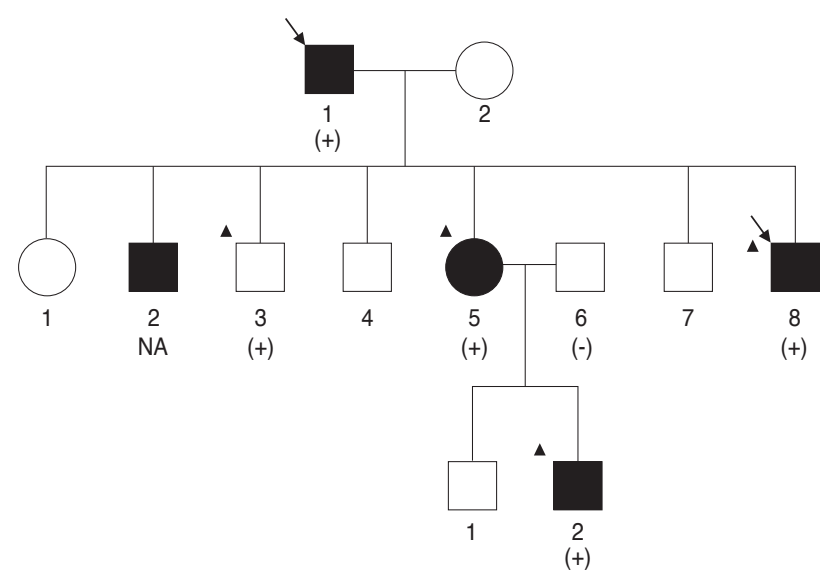

Fig. 1. Pedigree of the extended family. Circles represent females and squares represent males. Black symbols indicate affected individuals and white symbols indicate unaffected individuals. We performed a genetic analysis of five affected individuals (I-1, II2, II-5, II-8, and III-2) and two other family members (II-3 and II$6)$. Those with a mutation are indicated with $(+)$; the mutation is indicated with an arrow. Those without mutations are indicated with (-). Patients with the c.1362 1364insCGG mutation are indicated with an arrowhead. Patients with the c.1142_1144insGGC mutation are indicated with an arrow. $\mathrm{NA}=$ no amplification.
We received informed consent from all subjects before performing genetic analysis. This study was approved by the Catholic Medical Center Institutional Review Board (subject number SC13ZISE0164). Genomic DNA was extracted from each blood or buccal mucosal epithelial cell sample using a genomic DNA miniprep kit for blood (Axygen Scientific, Union City, CA, USA).

Polymerase chain reaction (PCR) was performed using $25 \mathrm{ng}$ of each genomic DNA template in a mixture of PCR buffer, which consisted of $2.5 \mathrm{mM} \mathrm{MgCl}_{2}, 200 \mathrm{nM}$ dNTPs, 0.4 pmol of each primer, and $0.75 \mathrm{U}$ of h-Taq polymerase (Solgent, Daejeon, Korea). For DNA sequencing, the amplified DNA was purified (QIAquick PCR purification kit; Qiagen, Hilden, Germany) and sequenced on a 3730xl automated DNA sequencer (Applied Biosystems, Foster City, CA, USA). The primers used for the genetic analysis of FOXC1 and PITX2 are shown in Table 1.

\section{Results}

\section{Case details}

\section{1) Patient I-1}

An 80-year-old male was referred to our hospital because of low vision in both eyes. His visual acuity had progressively decreased following an acute rise in intraocular pressure (IOP) about 25 years ago. At the first visit to our clinic, his right eye was unresponsive to light and his visual acuity in the left eye was limited to light perception. His IOP in the right eye was $10 \mathrm{mmHg}$ as measured by Goldmann applanation tonometry. His left eye was in a state of pthisis bulbi, and the fundus in the right eye was not observed because of a mature cataract. A gonioscopic examination revealed multiple patch-shaped iridocorneal adhesions at the superior angle. This patient's clinical features are summarized in Table 2 .

\section{2) Patient II-2}

A 60-year-old male was referred to our hospital because of low vision in both eyes. He had experienced an acute rise in IOP in his right eye about 15 years ago. His right eye did not perceive light, and his best-corrected visual acuity in the left eye was $20 / 100$. The IOP in the left eye was $15 \mathrm{mmHg}$, and he was using topical anti-glaucomatous medication (bimatoprost $0.01 \%$ ) in each eye. Slit-lamp ex- 
amination demonstrated phthisis bulbi with band keratopathy in the right eye, and overall iris atrophy and posterior embryotoxon at the temporal limbal area in the left eye. On gonioscopic examination, multiple patch-shaped iridocorneal adhesions were found at $360^{\circ}$ angles. The cup-todisc ratio (CDR) in each eye was 0.9. A Humphrey visual field 24-2 test showed severe glaucomatous visual field defects in the left eye. The central corneal thickness was 525 $\mathrm{nm}$ in the left eye. The patient's clinical features are summarized in Table 2.

Table 1. Primers used for the genetic analysis of FOXC1 and PITX2

\begin{tabular}{llc}
\hline & \multicolumn{1}{c}{ Primer $\left(5^{\prime} \rightarrow 3^{\prime}\right)$} & Amplicon $(\mathrm{bp})$ \\
\hline FOXC1_1F & GTCTGCTTTCCCCGTT & 564 \\
FOXC1_1R & TAGCTATAGGGCGGCTTCAC & \\
FOXC1_2F & ATGAGCGTGTACTCGCAC & 372 \\
FOXC1_2R & GGAAGCTGCCGTTCTCGAAC & \\
FOXC1_3F & AACGAGTGCTTCGTCAAGGT & 603 \\
FOXC1_3R & CAGAAGGCCGGAGCTGAG & \\
FOXC1_4F & ACCATAGCCAGGGCTTCAG & 517 \\
FOXC1_4R & CAGGTACCACGAGGTGAGG & \\
FOXC1_5F & CAAGCCATGAGCCTGTACG & 798 \\
FOXC1_5R & AGAAGAAACTTACGTGTTATCTGG & \\
PITX2 1F & TAACCTCTGGGCACTTTTGC & 572 \\
PITX2 1R & CTGGCGATTTGGTTCTGATT & \\
PITX2 2F & GCGGTGGACTAGGAAGCAG & 571 \\
PITX2 2R & CCAGAGGCGGAGTGTCTAAG & \\
PITX2-3F & GGCATGCTGACGGGAAAG & 300 \\
PITX2-3R & CTGTACCTCCACAACATCCTC & \\
PITX2-4F & TTTTTGGACCTGGAGAAACG & 976 \\
PITX2-4R & GCCCACGACCTTCTAGCATA & \\
FOXC1_ & & \\
\hline
\end{tabular}

$\mathrm{FOXC1}=$ forkhead box $\mathrm{C} 1$; PITX2 = pituitary homeobox 2.

\section{3) Patient II-5}

This 53-year-old female had a history of multiple ocular procedures, including retinal detachment surgery in the right eye and cataract extraction in the left eye. She was referred to our hospital because of decreased visual acuity, with the observation of glaucoma in the left eye. Her right eye was unresponsive to light, and her visual acuity in the left eye was $20 / 250$. Her right eye was in a state of pthisis bulbi. The IOP in the patient's left eye was 13 $\mathrm{mmHg}$ by Goldmann applanation tonometry. Slit-lamp examination revealed overall iris atrophy in the left eye. A fundus examination in the left eye showed a CDR of 0.9 and a pale optic disc. A Humphrey visual field 24-2 test showed a severe glaucomatous visual field defect in the left eye. The central corneal thickness was $407 \mathrm{~nm}$ in the left eye. Her ocular and systemic findings are shown in Table 2 .

\section{4) Patient II-8}

A 49-year-old male was referred to our hospital because of decreased visual acuity in the right eye. He had a history of cataract extraction in the left eye one year ago. His best-corrected visual acuity was 20/40 in the right eye and 20 / 32 in the left eye. He was using topical anti-glaucomatous medication (timolol 0.1\%/dorzolamide $0.04 \%$, brimonidine $0.15 \%$, and travoprost $0.004 \%$ ) in both eyes, and his IOP was $34 \mathrm{mmHg}$ in the right eye and $19 \mathrm{mmHg}$ in the left eye. Both corneas showed non-specific findings, and slit-lamp examination revealed overall iris atrophy in both eyes and an iris defect at 10-12 o'clock in the left eye. Fundus examination showed a $0.9 \mathrm{CDR}$ in the right eye and $0.85 \mathrm{CDR}$ in the left eye, with peripapillary atrophy and temporal and inferior neuroretinal rim thinning to the disc margin. On gonioscopic examination, both eyes had multiple patch-shaped iridocorneal adhesions. A Hum-

Table 2. Clinical features of the affected family members

\begin{tabular}{|c|c|c|c|c|c|c|c|c|c|c|}
\hline \multirow{2}{*}{$\begin{array}{l}\text { Pedigree } \\
\text { no. }\end{array}$} & \multicolumn{2}{|c|}{ Cornea } & \multirow{2}{*}{$\begin{array}{c}\text { Angle } \\
\begin{array}{c}\text { Iridocorneal } \\
\text { adhesions }\end{array}\end{array}$} & \multicolumn{3}{|c|}{ Iris } & \multirow[b]{2}{*}{ Glaucoma } & \multirow[b]{2}{*}{ Cataract } & \multirow{2}{*}{$\begin{array}{c}\text { Retinal } \\
\text { detachment }\end{array}$} & \multirow{2}{*}{$\begin{array}{c}\text { Systemic } \\
\text { abnormalities }\end{array}$} \\
\hline & Embryotoxon & $\begin{array}{c}\text { Band } \\
\text { keratopathy }\end{array}$ & & Hypoplasia & $\begin{array}{l}\text { Iris } \\
\text { hole }\end{array}$ & Corectopia & & & & \\
\hline $\mathrm{I}-1$ & $(-)$ & $(+)$ & $(+)$ & $(+)$ & $(-)$ & $(-)$ & $(+)$ & $(+)$ & $(-)$ & $(-)$ \\
\hline II-2 & $(+)$ & $(+)$ & $(+)$ & $(+)$ & $(-)$ & $(-)$ & $(+)$ & $(+)$ & $(-)$ & $(-)$ \\
\hline II-5 & $(-)$ & $(+)$ & $(+)$ & $(+)$ & $(-)$ & $(-)$ & $(+)$ & $(+)$ & $(+)$ & $(-)$ \\
\hline II- 8 & $(-)$ & $(-)$ & $(+)$ & $(+)$ & $(+)$ & $(+)$ & $(+)$ & $(+)$ & $(-)$ & $(-)$ \\
\hline III-1 & $(-)$ & $(-)$ & $(+)$ & $(+)$ & $(-)$ & $(-)$ & $(+)$ & $(-)$ & $(-)$ & $(-)$ \\
\hline
\end{tabular}


phrey visual field 24-2 test showed scattered scotomas in the right eye, and an arcuate scotoma in the left eye. The average RNFL thickness in the left eye was reduced upon optical coherence tomography examination, especially in the inferior quadrants. The central corneal thickness was $526 \mathrm{~nm}$ in the right eye and $517 \mathrm{~nm}$ in the left eye. The axial length was $23.69 \mathrm{~mm}$ in the right eye and $23.39 \mathrm{~mm}$ in the left eye. His ocular and systemic findings are shown in Fig. 2 and Table 2.

\section{5) Patient III-1}

A 19-year-old male developed glaucoma in adolescence and was referred to our hospital because of uncontrolled IOP. His best-corrected visual acuity was $20 / 32$ in the right eye and $20 / 25$ in the left eye. He was using topical anti-glaucomatous medication (timolol $0.1 \%$ /dorzolamide $0.04 \%$, brimonidine $0.15 \%$, and travoprost $0.004 \%$ ) in both eyes, and his IOP as measured by Goldmann applanation tonometry was $36 \mathrm{mmHg}$ in the right eye and $31 \mathrm{mmHg}$ in the left eye. Both corneas showed non-specific findings on slit-lamp examination. Gonioscopic examination revealed overall iris atrophy in both eyes, with multiple patchshaped iridocorneal adhesions in both eyes. Fundus examination showed a $0.9 \mathrm{CDR}$ in the right eye and $0.8 \mathrm{CDR}$ with neuroretinal rim thinning to the disc margin at 6 o'clock in the left eye. A Humphrey visual field 24-2 test showed a ring scotoma in the right eye and a superior nasal step visual field defect in the left eye. In both eyes, the average RNFL thickness was reduced, as was that of all quadrants and sectors except on the nasal side. The central corneal thickness was $527 \mathrm{~nm}$ in the right eye and $523 \mathrm{~nm}$ in the left eye. The axial length was $24.45 \mathrm{~mm}$ in the right eye and $24.10 \mathrm{~mm}$ in the left eye. We performed a trabeculectomy with mitomycin $\mathrm{C}$ in both eyes because of the uncontrolled IOP despite maximum tolerated medical therapy. Biopsy of the iris revealed degeneration in both eyes.
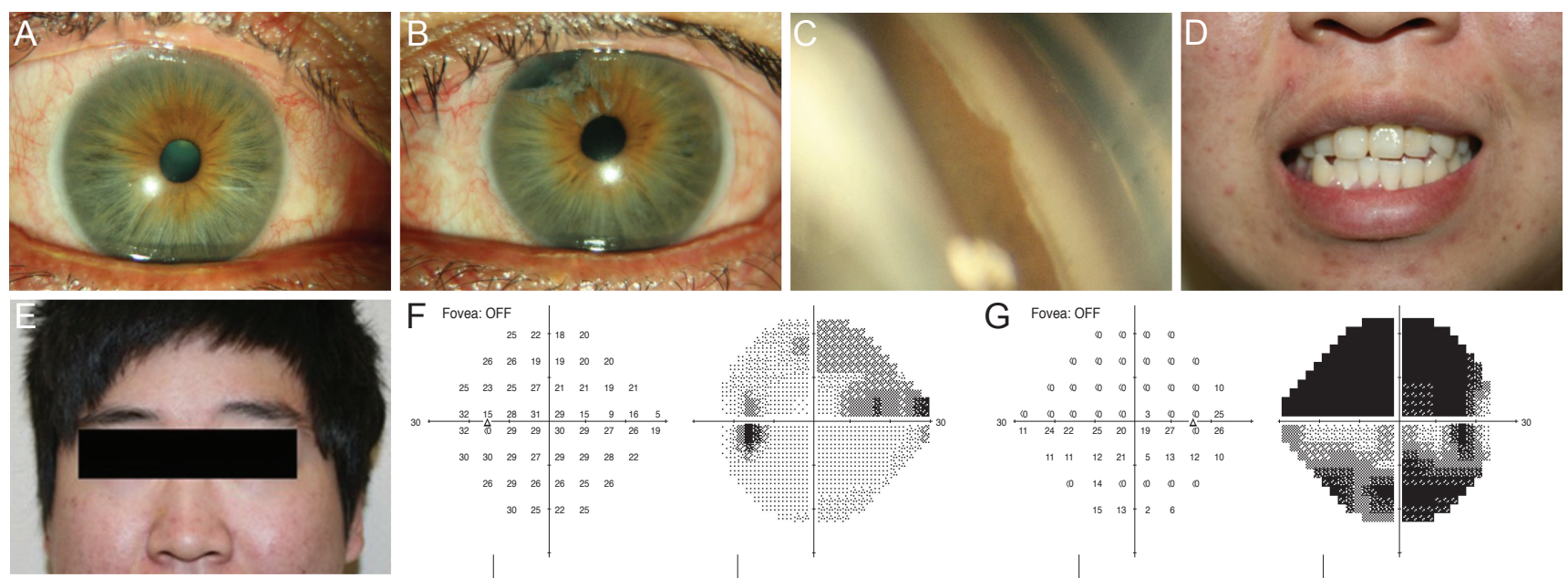

\begin{tabular}{lll|llll}
26 & 29 & 26 & 26 & 25 & 26
\end{tabular}

\begin{tabular}{ll|lll}
30 & 25 & 22 & 25
\end{tabular}
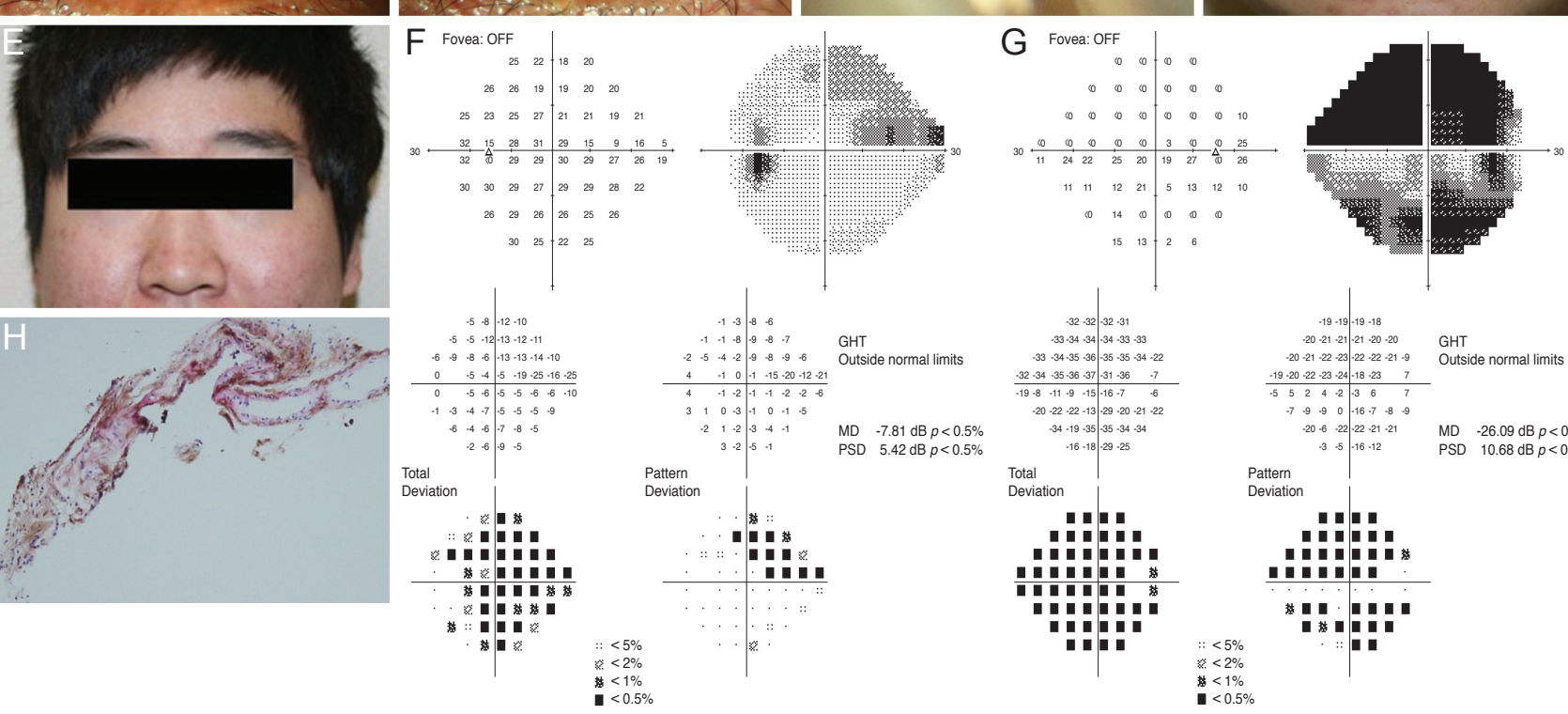

Fig. 2. (A) Patient II-8. Slit-lamp photograph of iris stromal hypoplasia with exposure of the sphincter muscle of the right eye. (B) Patient II-8. Slit-lamp photograph of iris atrophy and a defect at 10-12 o'clock in the left eye. (C) Patient II-8. Gonioscopy photographs of the iridocorneal angle showing broad iridocorneal adhesions. (D,E) Patient III-1. A physical examination revealed no facial abnormalities, including dental anomalies. (F,G) Patient III-1. A visual field examination showed a nasal step in the left eye and ring scotoma in the right eye. (H) Patient III-1. Histologic examination demonstrating iris degeneration $(\times 100)$. GHT = glaucoma hemifield test; MD = mean deviation; $\mathrm{PSD}=$ pattern standard deviation. 
At the fourth postoperative month, we performed needling revision and a 5-fluorouracil subconjunctival injection in his left eye because of Tenon cyst formation, accompanied by IOP elevation. The IOP in both eyes was observed in the range of 15 to $19 \mathrm{mmHg}$. The ocular and systemic findings for this patient are shown in Fig. 2 and Table 2.

A dental assessment was carried out in all affected members for the evaluation of craniofacial anomalies and dental abnormalities, including microdontia, hypodontia, oligodontia, taurodontia, enamel hypoplasia, and abnormally shaped teeth (particularly peg-shaped teeth). This assessment gave nonspecific findings. Other systemic abnormalities were denied by all affected members of the family during clinical interviews.

\section{Genetic analysis of PITX2 and FOXC1}

We obtained peripheral blood samples from three affected members of the family: the proband, a 19-year-old male (III-1); his mother, a 53-year-old female (II-5); and a maternal uncle, a 49-year-old male (II-8). We also obtained buccal mucosal epithelial cells from other affected members of the family: the proband's maternal grandfather, an 80-year-old-male (I-1), and another maternal uncle, a 60 -year-old-male (II-2). Buccal mucosal epithelial cells were also collected from two unaffected members of the family as control subjects: another maternal uncle of the proband, a 59-year-old-male (II-3), and the proband's father, a 58-year-old-male (II-6).

We performed DNA sequencing on all samples. Four affected members manifested two heterozygous mutations in FOXC1 and none in PITX2. Patients II-5, II-8, and III-2 had the FOXC1 mutation c.1362_1364insCGG. Patients I-1 and II-8 had the FOXC1 mutation c.1142_1144insGGC. Patient II-2's DNA could not be amplified because of the small size of the specimen. DNA from one of the controls (II-6) had no mutations. The other control (II-3) had the FOXC1 mutation c.1362_1364insCGG. The DNA sequencing results for FOXC1 are shown in Fig. 3. Both mutations result in amino acid changes and are frameshift mutations: c.1362_1364insCGG leads to p.Gly452fs, and c.1142_1144insGGC to p.Gly379fs. The affected patients were heterozygous for these two mutations, which occurred in the coding region for the evolutionarily conserved forkhead domain.

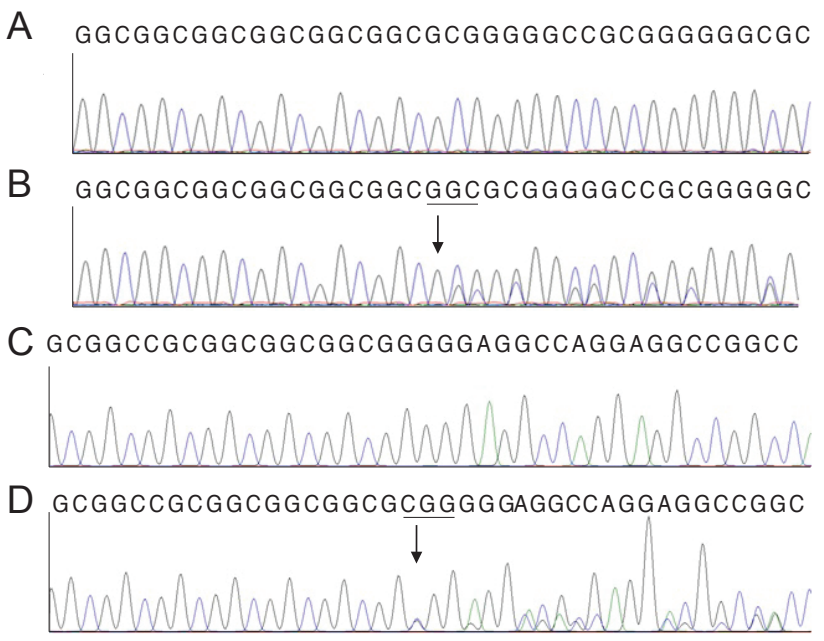

Fig. 3. DNA sequence analysis of the forkhead box $\mathrm{Cl}$ gene. (A, C) The reference sequences derived from a normal subject are shown. (B) The sequence derived from ARS patients I-1 and II- 8 shows a heterozygous mutation c.1142 1144insGGC, which results in a p.Gly379fs frameshift mutation. (D) The sequence derived from Axenfeld-Rieger syndrome patients II-5, II-8, and III2 and from family member II-3 shows a heterozygous mutation c.1362_1364insCGG, which results in a p.Gly452fs frameshift mutation.

\section{Discussion}

Patients with ARS are at approximately a 50\% risk for the development of glaucoma, with a greater risk in cases where the peripheral iris is highly attached to the trabecular meshwork. ARS results from a developmental arrest of the anterior segment occurring during gestation. This developmental arrest causes incomplete posterior recession of the posterior uvea, and the anterior uvea is highly attached to the trabecular meshwork. Both the trabecular meshwork and Schlemm's canal develop incompletely. The developmental arrest results in the partial retention of primordial endothelial cells on the iris and in the iridocorneal angle. The retained endothelium creates iridocorneal strands and their contraction leads to changes in the iris, including corectopia and an iris hole [1,2]. All affected members in the case family had developed glaucoma in young adulthood.

Our patients presented with posterior embryotoxon, iris hypoplasia, iridocorneal adhesions, and advanced glaucoma in the clinical ophthalmic evaluation. No systemic abnormalities were identified during the clinical interviews, and a dental assessment carried out on all affected members found no dental or craniofacial anomalies. 
In our case, four affected members of a family showed a mutation in the $\mathrm{FOXC1}$ gene, affecting the coding region of the evolutionarily conserved forkhead DNA-binding domain. To date, 46 cases of mutations in FOXC1 in people with ARS have been reported [7]. Recently, a novel c.317delA mutation in FOXC1 was detected in a Korean family [8]. Our case family presented with two mutations. One, c.1362_1364insCGG, results in a frameshift and amino acid change, p.Gly452fs. The other, c.1142_1144insGGC, also results in a frameshift and amino acid change, $p$. Gly379fs. Patients II-3, II-5, II-8, and III-2 in our case family had the same mutation, c.1362_1364insCGG, but each member showed a different phenotype. Iris hole was only manifested in patient II-8. Retinal detachment was only manifested in patient II-5. Even patient II-3 had no history of ocular surgery and does not have glaucoma. Patients I-1 and II-8 share the c.1142_1144insGGC mutation. Patient I-1 developed phthisis spontaneously.

Anterior segment dysgenesis disorders are heterogenous. In addition to the FOXC1 and PITX2 genes, several reports have linked other chromosomal loci to the development of the anterior segment, including 13q14 and 16q24, although the definitive causative genes have not been identified [7,9]. In addition, an association between PAX6 and ARS has been reported [10]. The CYP1B1 gene, located within the GLC3A locus, encodes a human cytochrome p450 enzyme, and its mutation has been reported to interrupt normal anterior segment development, especially in cases of primary congenital glaucoma. Recently, a case was reported in which a CYP1B1 mutation caused primary congenital glaucoma accompanied by features of ARS, including a broad nasal bridge and redundant periumbilical skin [11].

The PAX6 gene mutation causes both Peters' anomaly and aniridia and a CYP1B1 gene mutation results in Peters' anomaly and primary congenital glaucoma [10,11]. Even though we do not describe it in this report, our mutations are also found in patients diagnosed with Peters' anomaly in our clinic. It has also been reported that a point mutation (Phe112Ser) in FOXC1 causes ARS and Peters' anomaly in a single family [12]. Additionally, a study has shown that patients with the same mutation can manifest variable clinical findings, including no abnormal findings [13].

FOXC1 gene expression has been strongly detected in skeletal muscle, kidney, liver, and heart [6]. Forkhead factors have been reported to play a fundamental role in embryogenesis and in the expression of tissue-specific genes [14].
For this reason, systemic abnormalities have been occasionally reported in cases diagnosed with ARS resulting from mutations in FOXC1. The most common types of systemic abnormality are heart defects and hearing loss. Dental anomalies have also been reported in ARS $[6,15]$.

There is not a sufficient number of cases to prove the genotype-phenotype correlations in ARS. Strungaru et al. [15] studied 126 cases of ARS and showed that individual types of mutations lead to different clinical manifestations and prognoses for glaucoma. In their study, PITX2 defects mainly cause polycoria and systemic abnormalities. Meanwhile, in most cases, FOXC1 mutations result in ocular abnormalities (e.g., posterior embryotoxon, iridocorneal adhesion, iris hypoplasia, or corectopia) but relatively few systemic abnormalities in comparison with PITX2 defects. FOXC1 duplication is manifested by iridogoniodysgenesis, and has a more severe prognosis than other mutations [15]. A similarity between their study and ours is that the FOXC1 mutations showed no systemic abnormalities.

In summary, we describe variable clinical findings in a single family with Axenfeld-Rieger anomaly in this report. They share variable abnormal ocular findings and have no systemic anomalies. Patients with ARS have different clinical features, and the development of glaucoma depends on variable mutations and environmental stresses. We performed DNA sequencing on the FOXC1 and PITX2 genes, and two FOXC1 mutations were identified in our family. These mutations have not been reported in other studies. Although more functional studies will be needed to prove the association between these mutations and ARS, these mutations are new candidates that should be screened for in determining the risk for anterior segment dysgenesis.

\section{Conflict of Interest}

No potential conflict of interest relevant to this article was reported.

\section{References}

1. Shields MB. Shields textbook of glaucoma. 6th ed. Philadelphia: Lippincott Williams \& Wilkins; 2011. p. 227-35.

2. Shields MB. Axenfeld-Rieger syndrome: a theory of mechanism and distinctions from the iridocorneal endothelial 
syndrome. Trans Am Ophthalmol Soc 1983;81:736-84.

3. Alward WL. Axenfeld-Rieger syndrome in the age of molecular genetics. Am J Ophthalmol 2000;130:107-15.

4. Anderson DR. The development of the trabecular meshwork and its abnormality in primary infantile glaucoma. Trans Am Ophthalmol Soc 1981;79:458-85.

5. Challa P. Glaucoma genetics. Int Ophthalmol Clin 2008;48: 73-94.

6. Pierrou S, Hellqvist M, Samuelsson L, et al. Cloning and characterization of seven human forkhead proteins: binding site specificity and DNA bending. EMBO J 1994; 13:5002-12.

7. Phillips JC, del Bono EA, Haines JL, et al. A second locus for Rieger syndrome maps to chromosome 13q14. Am J Hum Genet 1996;59:613-9.

8. Kim GN, Ki CS, Seo SW, et al. A novel forkhead box C1 gene mutation in a Korean family with Axenfeld-Rieger syndrome. Mol Vis 2013;19:935-43.

9. Pal B, Mohamed MD, Keen TJ, et al. A new phenotype of recessively inherited foveal hypoplasia and anterior segment dysgenesis maps to a locus on chromosome 16q23.224.2. J Med Genet 2004;41:772-7.
10. Riise R, Storhaug K, Brondum-Nielsen K. Rieger syndrome is associated with PAX6 deletion. Acta Ophthalmol Scand 2001;79:201-3.

11. Tanwar M, Dada T, Dada R. Axenfeld-Rieger syndrome associated with congenital glaucoma and cytochrome P4501B1 gene mutations. Case Rep Med 2010 Aug 9. http:// dx.doi.org/10.1155/2010/212656.

12. Honkanen RA, Nishimura DY, Swiderski RE, et al. A family with Axenfeld-Rieger syndrome and Peters Anomaly caused by a point mutation (Phe112Ser) in the FOXC1 gene. Am J Ophthalmol 2003;135:368-75.

13. Ito YA, Walter MA. Genomics and anterior segment dysgenesis: a review. Clin Experiment Ophthalmol 2014;42:1324.

14. Larsson C, Hellqvist M, Pierrou S, et al. Chromosomal localization of six human forkhead genes, freac-1 (FKHL5), -3 (FKHL7), -4 (FKHL8), -5 (FKHL9), -6 (FKHL10), and -8 (FKHL12). Genomics 1995;30:464-9.

15. Strungaru MH, Dinu I, Walter MA. Genotype-phenotype correlations in Axenfeld-Rieger malformation and glaucoma patients with FOXC1 and PITX2 mutations. Invest Ophthalmol Vis Sci 2007;48:228-37. 